\title{
Les deux noms de F. J. Temple
}

\author{
Bernard Fournier
}

Paris

T. e suis un arbre voyageur/ mes racines sont des amarres// Si le monde est mon océan/ en ma terre je fais relâche// Ma tête épanouit ses branches/ à mes pieds poussent des ancres// Loin je suis près des origines/ quand je pars je ne laisse rien/ que je ne retrouve au retour ${ }^{1}$. » La double métaphore maritime et végétale rend compte de la double postulation du poète : d'un côté la terre, " ma » terre, précise-t-il, de l'autre, la mer qui s'offre aux pieds de la ville pour l'inviter aux explorations. Relevons aussi la surprise de l'enjambement des deux derniers vers : "Quand je pars je ne laisse rien/ que je ne retrouve au retour ", qui rejoint l'oxymore du vers précédent, "loin je suis près des origines ". Par cette figure de style, le poète tente de réunir dans une même voix les deux sources qui constituent son être : le sol natal et ses voyages.

De cette double orientation naissent deux noms donnés au poète par des "hommes-frontières", double orientation qui situe résolument la poésie de Temple dans une aventure ontologique.

Le paysage natal forme l'enfant, et la lecture l'invite au voyage imaginaire. Les pays et les gens que le poète rencontre vont imprimer de nostalgie tous ses périples et pousser le poète au lyrisme. Le regret d'un Passé défi $n i$, pour reprendre un titre de Cocteau, amène le poète à se considérer luimême, à se construire, et dans cette fuite, et dans ce retour, à travers les autres, à travers les femmes et même jusqu’à Dieu.

1 La Chasse infinie, éd. Granit, coll. De la Clef, 1995, p. 25, rééd. avec un frontispice de Claude Viallat, Jacques Brémond, Rémoulins sur Gardon, 2004; repris in Poèmes, traduit en occitan par Max Rouquette, Jorn, Montpeyroux, 1999, p. 63. 


\section{Les lectures, la culture}

A) UNE TERRE

Tous les livres de Temple insistent sur un point précis, donné en quatrième de couverture : l'auteur est né et vit à Montpellier. Ses racines sont là, dans ce Languedoc méditerranéen, comprimé entre la mer et le causse. Le recueil Villages du sud en est le manifeste, qui ajoute à la liste des villages et des lieux-dits, une litanie, un hymne : "Villages en sueur [...]/ Villages crissants $[\ldots]$ / Villages de lagunes $[. .$.$] / Villages qui sentent l'amour [\ldots] /$ Villages où se répandent des soleils chargés de vins, village de tourdres $[\ldots] /$ Villages où l'homme se mesure à lui-même, se reconnait, s'honore, se parle et parle à ses racines " $^{2}$. Cet appel, aux « villages », sur le mode vocatif, donne au poème des tons de prosopopées qui nous font littéralement voir ces pays du sud, assommés de soleil, pays que le poète connaît d'un amour intime et où il reconnaît ses racines. Et la liste des villages apporte une pointe d'accent au phrasé de Temple : Saturagues, Fabrègues, SaintGuilhem-le-désert, Mèze, Vic-la-Gardiole, Maguelone, Saint-Georgesd'Orques, Sainte-Croix-de-Quintaillargues, Frontignan, Bouzigues, Castelnau-le-ne, Villeneuve-lès-Maguelone, Sommières, Puéchabon, Clapiers, Palavas, Guzargues, Le Grau-du-roi, Argeliers, Balaruc-le-vieux. Nommer, c'est s'inscrire dans le monde, dans son monde et le poète s'installe, s'ancre dans son pays par l'écriture. C'est son port d'attache, là où son premier nom lui fut donné.

Et c'est " d'outre terre " que sont venus les peuples de l'Aubrac: " emportés par le vent du destin/ vers ces noires landes jaunes/ où des oiseaux démesurés/ ont dans la pénombre des temps/ déposé leurs œufs de granit " (La Chasse infinie, 36). Les images que nous donne le poète de son pays sont incomparables. Tout y parle de l'origine, des hommes et des animaux. Le poète crée une mythologie personnelle qui fait venir les pierres levées de l'Aveyron d'un univers fantastique. "Où suis-je? " se demande-til. Pour répondre : "C'est mon pays ", avant de tenter un autoportrait en homme des causses : "Cet homme qui ressemble à la terre,/ peau d'écorce, chair d'aubier,/ jambes de racines torses,/ oint du musc des troupeaux,/ qui marche toujours sur les sentes/ où mugit la conscience perdue/ dans la rumination des siècles,/ c'est moi. » (La Chasse infinie, 36-37) L'homme se confond non seulement à la terre, mais aussi aux animaux, aux arbres, pour retrouver des origines intemporelles.

2 Anthologie personnelle, Actes Sud, Poésie, 1989, p. 157. 


\section{B) LES DEUX ONCLES}

Retenons cette formule " oint du musc des troupeaux » : on a là un véritable baptême de sueur, d'intime relation avec les animaux. Baptême auquel feront échos deux autres que recevra le poète. Temple se peint en paysan du Larzac, terre aride et dure, froide en hiver, chaude en été, hautes terres par leur altitude et leur origine volcanique qui les font remonter aux origines de la formation de la terre. Ce paysan devient alors comme les animaux dont il est le frère, issu de la terre, venant d'elle comme une autre racine.

Je reprends à dessein cette formule associée à Cendrars (Le Panama...). Ces deux oncles représentent pour l'enfant des pères spirituels, ceux qui vont le guider, ceux qui vont l'amener à un second baptême.

Le premier de ces oncles, en fait un grand-oncle, ne vient que sur le tard dans la vie du poète, au hasard de la découverte d'un journal intime que cet aventurier rédigea sans le publier : " un oncle barbu qui avait franchi à dos de lama les frontières du royaume inca, chassé l'anaconda avec un chien malade dans les marécages de l'Argentine, enjambé les corps endormis des gauchos dans les corridors nocturnes des posadas sous le regard mauve de navajas piquées dans le plancher " ${ }^{3}$. Par l'accumulation, le poète donne à voir un aventurier aux multiples souvenirs. L'exotisme des mots relève de cette propension à faire rêver le lecteur.

Cet oncle « avait ramené dans ses valises des sacs de cuir bourrés de serpents mortels, de petits coralles vermillon, des mocassins, des jararacas prolifiques des surcucus capables de foudroyer un régiment de bothrops-ferde-lance dont le venin arrête les hémorragies et aussi de noirs mussuranas inoffensifs; cet oncle avait voyagé avec les derniers 'hommes-frontières'». (Les Eaux mortes, 74-75) L'image de cet homme produit un effet certain sur celui que fascinent l'Amérique, sa faune, sa flore, et ses habitants, les «hommes-frontières ».

L'autre oncle apporte avec lui l'attirance vers l'Afrique. "Tandis qu'il piochait l'oncle Blaise me parlait du désert des méharées, à travers le Sahara, du Tibesti à la Mauritanie, couchant sous la tente avec les Touaregs ». (L'Enclos, 64) Ces Touaregs, précisément des " hommes-frontières ». Frontières de l'espace, mais aussi frontières du temps.

On peut ainsi remonter plus haut dans la pérégrination temporelle, et cet oncle s'y emploie, très concrètement dans ses activités d'archéologue auxquelles il fait participer son neveu : "Peu à peu la tombe était dégagée et c'était bientôt le moment, qui valait des siècles, de soulever la pierre plate

3 Les Eaux mortes, Albin Michel, 1975, Babel, Actes sud, 1997, p. 74-75. 
qui recouvrait l'ancêtre. Celui-ci gisait dans une terre qui conservait aussi ses armes, pointes de flèches pédonculées, à barbelures, dentelées ou crénelées, en feuilles de laurier, pointes de lance, éclats de silex, perles de jade, de gypse, d'ambre, de schiste, pendeloques, alènes d'os ou de métal, tessons, dents d'animaux, mêlés à la pierraille, aux racines des buis et des cades. Il était là, le vieux guerrier, avec une petite pointe de silex finement taillée, incrustée dans son tibia, enterré pour l'éternité. Non pas pour l'éternité. Nous étions là, l'oncle Blaise et moi, dans la contemplation d'un crâne qui regardait, du gouffre noir de ses yeux, ces intrus qui avaient interrompu l'absence du temps. Depuis six ou sept mille ans, il ne nous attendait pas. Mais si $\mathrm{j}$ 'avais alors connu le poème, $\mathrm{j}$ 'aurais pu l'invoquer : "pères profonds, têtes inhabitées ". J'avais peut-être devant moi mon très lointain arrière-grand-père, si lointain mais si proche, un contemporain, si l'on pense aux deux cents millions d'années des limules. " (Le Chant..., 3637) On devine l'importance de cette racine, au double sens du terme: d'un côté une lignée héréditaire revendiquée; de l'autre une appartenance au sol, à la terre. Comme si les morts ne s'enfonçaient pas dans l'humus, mais qu'au contraire, ils y prenaient naissance. C'est précisément cette nativité qu'y reconnaît le poète. La découverte a de quoi impressionner le jeune garçon, qui trouve là une de ses racines, une origine. Celle-ci n'entre pas en concurrence avec le chant des limules, d'où, parallèle à la joie de découvrir un père, un sentiment de déception autant que d'étrangeté, voire de viol.

\section{C) Les LeCtures}

C'est pourquoi le refuge dans la littérature demeure une source moins ambiguë que la recherche de soi.

Les écrivains de Temple se classent deux parties : d'un côté, ceux qui voyagent, ceux de la terre; de l'autre ceux qui questionnent le langage dans l'aventure de l'être.

Dans la première catégorie, ceux du voyage, nous rencontrons d'abord Jules Verne nommé étonnamment : « le plus grand poète des deux derniers siècles » (Le Chant..., 60), Puis vient Hérédia : "Vers quels Cipango n’allais-je pas, vers quels Orients »(Les eaux mortes, 14), qui renvoie bien évidemment aux "Conquérants ", poème évocateur s'il en fut. On passe ensuite aux vrais voyageurs : le voyageur de luxe que fut Valery Larbaud : « Le train qui m'emporte vers le terminus de l'Europe/ s'arrête parfois dans des gares béantes de feu/ [...] ce jeune homme gras ressemble à Barnabooth/ dans la moiteur je rêve à Fermina Marquez " (Anthologie..., 151) Le voyage lui-même est un palimpseste : il s'agit de mettre ses pieds

\section{LittéRéalité}


dans les pas de ceux qui nous ont précédés. Ainsi, c'est par l'intérêt porté à l'auteur de Childe Harold Pilgrimage que Temple a écrit l'histoire de la fille de Lord Byron, Médora, enterrée en Aveyron. On voit comment le poète s'attache à retrouver chez les écrivains tout ce qui se rapporte à eux, à leurs œuvres. Cette tombe, encore une fois dans " sa " terre d'Aveyron, le relie deux fois au poète.

À côté des écrivains proprement voyageurs, l'œuvre de Temple est traversée par ceux qui sont attachés à la terre. Ainsi Pline le Jeune (Les Eaux mortes, 141-43), dont il fait une large citation à propos de l'éruption du Vésuve. Chateaubriand est l'objet quant à lui d'un pèlerinage à "Combourg " : "Il était là, ce cœur/ à l'écoute des arbres/ et du temps vaporeux.// La douce souvenance " (Anthologie..., 119). La forme emphatique de "Il était là, ce cœur " montre à quel point Temple est sensible au lieu habité par l'écriture.

Notre deuxième axe comprend les écrivains qui intéressent Temple dans leur rapport avec leur questionnement ontologique. Shakespeare, notamment, avec la célèbre citation tirée d'Hamlet: " tre ou ne pas être [...] ne cherchez pas ici/ ce que savent les arbres/ et l'eau claire de l'Avon " (Anthologie..., 122). La réponse à l'interrogation doit venir des éléments. "Etre ", c'est la raison qui le pousse à venir au-devant des poètes, sur leur terre, pour les connaître davantage, chaque écrivain étant pour le poète comme pour lui-même l'homme d'un lieu. Et c'est en voisin qu'il est sensible à l'aventure spirituelle de Joë Bousquet : "Traduit du silence, écrit par un homme qui vivait la nuit, cloué au lit depuis sa guerre » (Les Eaux mortes, 110). Le nom n'est pas cité, comme si seul le titre devait porter en lui toute la dimension de cette entreprise.

Il faut enfin mentionner les écrivains que Temple a connus et dont il tient à découvrir les lieux de leur vie et de leur livre, et dont, paradoxalement, nous ne dirons rien, tentant de rester bref: ils font partie d'une autre anthologie, celle du vécu : Blaise Cendrars, Lawrence Durrel, D.H. Lawrence, Henry Mller, Joseph Delteil, David Gascoyne, Richard Aldington.

On voit comment ces lectures demeurent une nourriture essentielle, existentielle, comment elles le relient à la terre et comment tout à la fois elles l'en éloignent pour mieux la retrouver. Elles mèneront l'adulte à parcourir, sinon le monde, pour le moins les lieux dont il a rêvé, et qui ont fondé sa naissance.

La terre occitane, deux oncles et des lectures ont marqué à jamais cet homme qui va devenir ce voyageur à la recherche de sa propre identité. 


\section{Le chant du voyageur}

Le voyage chez Temple s'inscrit dans une démarche existentielle qui prend appui sur la littérature. Mais le départ demeure toujours autant un arrachement qu'un espoir, arrachement à sa terre et à soi; espoir de découvrir d'autres terres et d'autres mois; c'est pourquoi le chant de Temple se teinte d'une grande nostalgie tournée vers l'être qu'il fut.

\section{A) LeS VOYAGES}

Le voyage appelle le poète aussi bien qu'il l'invoque et le convoque par de grands élans lyriques : "À la pleine lune sonnez du cor [...]// Nous proclamerons les Noms et les Nombres/ qui marchent depuis le commencement/ au souvenir des paroles premières [...]// Sonnez du cor dans les villes mouvantes [...]// Sonnez du cor levez-vous dans l'aurore/ qui nous revêt d'iris et de safran/ pliez les peaux affaitez vos montures/ il faut partir vers les herbes naissantes/ où l'ancienne mémoire nous attend. " (Anthologie, 183-84) Ces voyages vont éprouver leur nécessité pour celui qui part à la recherche de l'origine. D'où une joie d'enfant qui émane de leur relation poétique.

Le Nouveau Monde attire le poète : "J'étais à Nantucket. Nantucket existait. J'existais donc, puisque j'étais à Nantucket, dans le gel de décembre, buvant du thé au rhum avec Grant et Orland à la Pollard Tavern, tandis que des escouades de pétrels glapissaient en se jouant de la galerne... " (L'Enclos, 88-89) On voit quelle conscience a le poète de sa propre existence face à ses mythes. Il ancre dans le réel cette seconde patrie littéraire à force de noms propres, tandis que l'évocation de la nature renvoie davantage à un lien commun avec les lecteurs. C'est à partir de la réalité et non des lieux communs que Temple décrit ce qu'il voit où il se trouve : "Loiseau rouge/ du New Jersey/ Lance son cri/ dans les sumacs. " (Anthologie..., 129) «Tu lances ta ligne/ dans le Potomac/ et tu souris.// Nous allons rêver/ Vers Chesapeake Bay.// Nous buvons 'Chez Odette'/ du vin rouge de Californie/ en parlant de femmes/ et de Beiderbecke. » (Anthologie..., 129) Même si le lieu est commun, telle ou telle auberge, il est mythique par son nom : le Potomac, Cheasepeake Bay.

"À vrai dire, elle [l'Amérique] m'appartenait depuis ma plus lointaine enfance. Plongé dans les lectures, à l'Enclos, aurais-je pu imaginer que mon 'rêve américain' continuerait d'en être un en se réalisant? " (L'Enclos, 83) C'est ce qui apparaît de plus fort chez Temple : il est parvenu à inscrire le mythe dans la réalité. 
Et l'on voit bien que c'est encore l'enfance qui constitue le pôle central de la recherche du poète, d'où une grande nostalgie.

\section{B) Nostalgie}

Le voyage semble d'abord une fuite de soi, de l'être dont le nom n'est encore qu'emprunté; la raison même du voyage, comme pour Baudelaire, et c'est alors le voyage lui-même qui va le susciter, quand l'homme se construit par ses actes.

Il ne s'agit pas ici d'une simple nostalgie, celle que ressent l'homme à l'évocation de ses jeunes années. Chez Temple, ce sentiment remonte très haut et très loin, puisqu'il cherche à découvrir l'enfant qu'il fut, l'être qu'il est : « Comment donc puis-je m'intéresser à l'enfant de cinq ans qui porte mon nom mais dont l'homme qui a tenté de le retrouver est tout aussi éloigné que je le suis maintenant de lui ?// Nous ne sommes que des aliénés. Il n'est que de nous réfugier dans la seule réalité de notre propre mythe » (L'Enclos, 72).

Le voyage, en précipitant et le temps et l'espace, participe de cette construction à rebours : "Je me suis perdu sur la terre/ Sur chaque rivage un peu $[\ldots] / /$ Je me suis perdu, chaque escale/ Me fut tombe $[\ldots] / /$ Je me suis perdu dans le feu des longitudes $[\ldots] / /$ Je me suis perdu un peu partout où le vent pouvait emporter une voix humaine $[. ..] / /$ Je me suis perdu dans le monde " (Anthologie..., 64). La litanie met bien en valeur la perte de soi dans le monde, une sorte de décomposition progressive.

La nostalgie, malgré son chant élégiaque, n'abandonne pas sa conscience, sa distance qui peut aller jusqu’à la dérision : "Quelque chose meurt en moi [...]// O lune, ris de la rose noyée! " (Anthologie..., 61) Le voyage se double ainsi souvent de la mémoire qui la fait naître. En même temps, on le voit bien, on s'emporte toujours soi-même dans ses voyages, on ne recherche que soi, alors même qu'on veut découvrir autre chose pour se libérer. "Pourquoi le passé est-il si lourd? " se demande le poète un peu plus loin. "Nous voici désormais condamnés aux mirages/ à l'herbe amère des anciens jours" (Anthologie..., 147). Le chant résonne comme une élégie douloureuse qui n'a plus d'horizon que celui d'où il était parti.

Ainsi le voyage se double-t-il d'un sentiment ambivalent. D'un côté le plaisir du voyage lui-même, de la découverte des lieux de ses lectures, mais de l'autre il y a cette mémoire de l'enfance : "Je ne sais joie plus douloureuse que la joie des souvenirs répandus/ Celle des visages. [...] Je ne connais joie plus douloureuse que la vue des souvenirs répandus aux flancs légers des valises désertes. " (Anthologie..., 66) Ces deux vers enfer- 
ment dans leurs rets tout un poème, douloureux de l'impossibilité de dire : "Qui me dira ce soir la musique des cordages/ Et celle enivrante des hasards/ Gigantesques. [...] Ah, si je pouvais dire quelle musique s'élève de terre aux quatre coins du monde [...] Ah, qui me dira ce soir la musique étrange des forêts pleines d'ilang-ilang. " Le voyage ne renferme dans son souvenir que la nostalgie de n'être pas là, la nostalgie du temps et de l'espace qui se séparent. On en revient toujours vides de paroles non dites, d'hommes trop vite entr'aperçus, de paysages qu'on n'a plus sous les yeux, d'un peu de soi qu'on a laissé dans les ports d'attache.

Alors commence "le chant terrible de la mémoire " (L'Enclos, 114) qui doit redonner au poète par la force des mots un autre nom que le sien.

\section{C) LE LYRISME}

La poésie de F. J. Temple est marquée par un profond lyrisme qui semble paradoxal chez cet homme par ailleurs pudique et discret.

Ainsi nous avons souvent des exclamations : « donne-moi ta mémoire, $\mathrm{O}$ désert!"(Anthologie..., 142) Des reprises de vers qui rythment le poème : "J'ai pour toi préparé $[\ldots] /$ J'ai dessiné $[\ldots] /$ Je donnerai $[\ldots]$ " (Anthologie..., 142) Reprises qui sonnent parfois comme des litanies, ainsi les incontournables " j'ai vu ", propres aux voyageurs : "Et moi aussi j'ai pris la diligence $[\ldots]$ / J'ai vu les sauges grises " (Anthologie..., 132), "Je suis venu par le 'California Zephyr' [...]/ J'ai vu l'aube peindre en rose/ Les hautes cimes de la Sierra Nevada ». (Anthologie..., 133)

La forme de la litanie peut prendre des accents de douleur face aux paysages qui le glacent, face aux souffrances du monde : « En moi la nuit aux yeux de fer/ Contre le cœur mauve des gloires,/ En moi l'Empire aux couronnes d'étain/ Sur les balafres du silence,/ en moi le sombre cri des plaines/ Où gît l'odeur aveugle des blessures "; et il continue : "Voici le coq enchaîné dans le sang [...]// Voici le coq en sa robe d'augure [...]// Voici l'urne où mûrit l'huile des marécages [...]// Voici les grandes herbes en mouvance [...]// Voici le cri du jour en tunique de rime." (Anthologie..., 47) Litanie du réveil vécu comme une mort, " mort de mon sommeil ».

De ce terreau de souffrances s'élève un poème lyrique, heureux de pouvoir proférer des odes : " et je chante/ les fleurs du silence " (Anthologie..., $36)$; "Je chante comme arbre à guitares [...]/ Je chante le sang noir des Sept Cités de Cibola [...] / Je chante Alvar de Nunez Cabeza de Vaca [...]/ Je chante rouge la mort de Sitting Bull [...]/ Je chante noir la mort illuminée de Sitting Bull [...]// J'enchante les oiseaux, les femmes de vos nuits 
[...]// Je ne suis pas d'ici, mais je chante. " Antienne reprise et allongée : "Je ne suis pas d'ici, mais je chante/ Le bruit triste des cuivres brisés. [...]// Je ne suis pas d'ici, mais je chante/ Les bronzes de sueur des assemblés chorales/ Dans les grandes aires de poussière. [...]// Je ne suis pas d'ici, mais je chante/ Le rude laboureur Abraham Lincoln,/ Ce Capitaine, O quel Capitaine!/ Mille chants, j'entends chanter l'Amérique/ J'entends chanter les barques sur les fleuves,/ Capitaine, O Capitaine,/ J'entends chanter l'Amérique dans ses plaines.// Je ne suis pas d'ici, mais je chante./ Je chante les oiseaux purifiés dans les savanes/ Et te salue, sur tes ergots de ciment. » (Anthologie..., 71-72) L'ode se gonfle à la hauteur de l'épopée à l'évocation des héros et des guerres.

Cependant, malgré le souffle épique qui gonfle ces vers, le sentiment de perte se fait toujours sentir par des interrogations répétées où l'on entend un aveu de souffrance : «Où donc le ciel puise-t-il ses déluges/ pour envahir les âmes démâtées? [...]// Où commence la mer, où le terme du ciel,/ Où le port apaisant de nos pensées sans voiles? 》 (Anthologie..., 32) Pour finir par une exclamation des plus lyriques : "O moi, blessé, noyé, perdu de gestes vains/ irai-je, en quel asile, abriter les tourments,/ penché sur les croix blêmes des absences? " (Anthologie..., 32) Ce cri d'un lyrisme douloureux est lancé à travers la mort, l'absence et la déperdition de soi.

Les voyages rêvés et vécus appellent une nostalgie profonde d'un être qui se perd dans le temps et l'espace. Seul le chant lui rend une identité parce qu'il vient du plus profond de lui.

\section{La recherche existentielle}

Nul n'est poète s'il n'est pas à la recherche de soi-même. Chez Temple, cette quête (cette Chasse infinie) est passée par la lecture, puis par son prolongement qu'est le voyage. Mais " Partir, c'est mourir un peu ", a dit un autre poètét. Dans cette œuvre, il s'agit de lutter contre la mort, de celui qu'on fut, de soi-même, des autres et du monde. De fait, il s'agit de se retrouver à travers sa naissance, le contact avec les femmes, et par elles, avec Dieu et les hommes qui lui donneront enfin un baptême.

4 Edouard Haraucourt (1857-1941) : " Partir, c'est mourir un peu,/ C'est mourir à ce qu'on aime./ On laisse un peu de soi-même/ en toute heure et dans tout lieu. ", Rondel de l'adieu, Choix de poésies, Seuil. 


\section{A) LA FEMME}

La femme octroie à l'homme un surplus de considération : " mes yeux te disent/ quand tu es là je deviens beau " (Anthologie..., 37). Elle est un miroir qui renvoie une image rêvée, socialisée, narcissique.

C'est ce qui rend les rapports amoureux parfois tendus à l'extrême : "Couteaux sur nos regards/ telle est la joie du désespoir » (Anthologie..., 33). La femme est une éternelle souffrance qu'exprime un nouvel oxymore : "Le feu repousse/ qui le cherche;/ j'ai refermé sur toi/ mes doigts vides » (Anthologie..., 34), après une figure proprement poétique dans la plus pure tradition de l'amour courtois : "Le temps me brûle immobile fuyard/ Est-ce l'amour l'ombre de ton regard// Est-ce l'amour cet ange des ténèbres// Est-ce l'amour cette cendre qui neige [...]// Je vis de mort où je meurs de désir ". (Anthologie..., 38) La multiplication des oxymores dit combien la relation féminine chez Temple est d'une grande complexité. L'interrogation, bien rythmée, sous forme de litanie, laisse le poète sans amour.

Mais, fort heureusement, la relation amoureuse n'est pas uniquement dite sur le plan de la souffrance. L'homme et la femme peuvent former un couple harmonieux, où les êtres ne font plus qu'un, le couple sans lequel aucun des deux ne peut vivre : "Qui t'arrache de moi/ m'arrache,/ vaincu, sans mémoire. " (Anthologie..., 36) L'amour permet ainsi de donner au poète une part de lui-même, celle précisément qu'il cherche. Hors la femme, l'homme est sans passé.

Il fait d'elle un beau portrait : "Une fleur inconnue [...]// Une femme inconnue, née d'elle-même/ sur le sable d'amour lointain,/ Une barque au ventre d'ébène/ Ouvre un silence de sommeil/ sur mes rêves d'adolescence. [...]// Et moi qui parle invisible et blessé. " (Anthologie..., 45) Ce "ventre d'ébène " nous fait immanquablement penser à la mère du poète jouant au violoncelle, seule image que nous laisse voir le poète, éparpillée ici ou là dans les textes de Temple ${ }^{5}$ : "par quel coup d'archet/ jaillie des mamelles de la lune,/ quatre cordes entre les cuisses/ le dieu Pan bourdonne en son antre ". (La Chasse infinie, 23) La mère est érotisée à la manière d'une femme et les images fournissent de puissantes envolées vers l'imaginaire, vers le sacré de l'origine : "Quand la nuit pénètre la mer/ luisant de mille ardoises/ ma mère est là/ dans l'odeur des alyssons/ et des lys de sables. " (La Chasse infinie, 23) Se surimposant à la paronomase très guillevicienne, (mer/mère) les jeux de mots et de sons, entre " alyssons " et « lys de sables ",

5 Notamment dans Le Chant des limules, op. cit., p. 26. 
provoquent le lecteur pour le renvoyer à une érotisation antérieure à la naissance, dont le lys est l'élément symbolique, le poète approche à la métaphysique.

$\mathrm{La}$ femme donne naissance à l'homme. Non pas la mère, mais la femme alter ego, l'objet d'amour, celle qui fait d'un enfant un homme : "Infatigable,/ je vais vers qui je fus./ Je porte en moi qui me donne la vie./ On me nommera homme/ à cause de toi. " (Anthologie..., 25) L'homme remonte l'origine par la femme, et plus haut encore vers Dieu.

\section{B) La mystique}

"Sur les sommets tabulaires de la Black Mesa, les divinités du désert devaient se pencher de leur balcon de basalte, comme on nous dit que faisaient les dieux de l'Olympe, au son de l'aigre flûte bergère et du tambour de peau de chèvre. Je n'évoque pas la Grèce par hasard. Ce pays, lointain, lui ressemble par la vertu qu'il a de vous persuader que les dieux sont bien là, dans la terre colorée, les roches aux silhouettes changeantes et les sombres forêts des montagnes. Dans ce coin d'Amérique, le temps s'est arrêté. Rien ne peut faire que l'esprit qui a présidé au commencement du monde n'y demeure. Les Indiens sont toujours là, immuables après mille et mille ans, dans leurs villages de terre sèche, avec enfants, dindons, maïs bigarré, haricots, courgettes, tabac, turquoise, et la silencieuse vitalité qui coule en eux comme le Rio Grande dans ses gorges profondes. Souvent j'ai cru voir, au milieu des danseurs empanachés de plumes, Achille ou Hector parés pour le combat; souvent j'ai croisé Nausicaa, ondulante et brune, à la jambe un peu lourde, puisant de l'éau à la citerne avec la jarre polychrome semblable à celle de Mycène ou de Cnossos. La vigne et l'olivier rejoignent le maïs, porteur d'une civilisation stoppée dans son élan, pétrifiée dans son silence. » (Retour..., 13) Dans ce passage il me semble lire pour la première fois un parallèle entre deux civilisations apparemment contradictoires. Le Nouveau Monde de Temple rejoint le monde archaïque dont nous sommes issus. Le poète retrouve ainsi, dans l'Ouest lointain, le Far-West, la renaissance du monde.

C'est pourquoi la poésie de Temple se nourrit de la figure christique. Ce Christ est un homme profondément chtonien : «C'est par les veines de la terre/ que vient Dieu,/ par les pieds qui sont racines/ dans l'humus et la pierre,/ vers les cuisses, l'aine humide/ et douce/ comme un herbage de varaigne,/ et non du ciel/ virginal/ où il ne trône pas. " (La Chasse infinie, 9) L'érotisation de cette évocation, celle de la mère rejoint par la métaphore 
du corps, "les veines de la terre", pour mieux assurer une vision humaine du Christ.

La Passion est revécue parce qu'elle concrétise la renaissance : «Voici mon corps, ce pain de paysan [...]/ Voici mon corps, orné de vomissures ». (Anthologie..., 15) L'élément nourricier est ici troublé par l'élément de rejet qui symbolisent à eux deux, dans une simplicité proche de la trivialité, la mort et la renaissance. Les paroles sont les mêmes que dit le prêtre lors de l'office : « Lorsque étrangère, en une ville de douleur,/ l'aube durcira tes paupières, / les douces larmes d'une pluie d'été/ te berceront de musiques lointaines,/ en mémoire de moi. " (Anthologie..., 39) Le poète redevient le prêtre qu'il était à l'origine du religieux, officiant d'un rituel culturel autant, ici, que personnel.

C'est pourquoi, la mort du Christ, c'est celle du poète : "Sachez que je suis déjà mort/ Moi qui vous parle [...]// Mais dans votre plus intime/ Elevez un tumulus/ à mon absence ». (Anthologie..., 57) Temple désire un rituel, une prière, une mémoire sous une forme archaïque, en dehors de la religion instituée. Il insiste ailleurs : "En vérité je suis mort [...]// J’allume un feu secret/ pour les rives du ciel/ dernier défi haute prière/ vers les dieux abolis. " (La Chasse infinie, 16) Comme le phénix, c'est au moment de cette mort symbolique que le poète acquiert toute son identité, une identité double qui correspond à sa double postulation de voyageur et de sédentaire.

\section{C) UNE RECHERCHE DE L'ÊTRE : LES DEUX NOMS.}

Le poète met en évidence les marques de cette seconde naissance que lui ont offerte les hommes dans ses voyages. Il y a acquis deux noms : un en Algérie, Dib, "l'Homme-qui-tue-le-chacal ", l'autre en Amérique, "Celuiqui-marche-dans-le-soleil $»^{6}$.

[V]oilà trente ans j'enroulais mon chèche autour de ma tête lorsque le vent soulevait le sable du désert, dans les terres salées du Sud-Oranais, dans les chotts, sous les eucalyptus du bivouac de Cassaigne, dans les gorges rouges des Aurès. Mon nom était Dib, 'le chacal', car j'avais l'art de dépouiller, sans répugnance aucune, ces bêtes pour le compte des Arabes de mon escadron; nous revenions chaque soir de nos expéditions de chasse avec deux ou trois

6 Retour à Santa Fe, voyage, éd. Proverbe, La Haute Solière, 61290 Marchainville, p. 34.

\section{LittéRéalité}


chacals que je pelais aussitôt sans attendre que la rigor mortis en ait durci les carcasses, et je clouais les peaux grasses et puantes sur les portes, sous l'œil aigu et respectueux de mes types qui m'avaient sacré Dib, l'homme-qui-tue-et-pèle-les-chacals, qui n'en craint pas l'abominable odeur; c'était tout juste s'ils ne répandaient pas le bruit que j'en dévorais la chair nauséabonde ». (Les Eaux mortes, 149-50) Un autre baptême lui est octroyé par les Indiens, les vrais, ceux de ses rêves, de ses lectures, qu'il a réussi à rencontrer, à être presque des leurs, vivant avec eux leurs rites et leurs coutumes : «Béni par les divinités qui dansent sur les montagnes, un nom me fut donné. J'eus des frères et des søurs. » (Anthologie..., 143) Ce nom n'est pas dit, par modestie, par déférence et par pudeur au cœur du lyrisme des vers : "Moitié espagnol; moitié anglais, il m’expliqua que les enfants m'avaient donné un nom. Je n'ai pu transcrire mon nom indien, mais je sais que depuis ce moment j'avais désormais à Taos des frères et des sœurs. J'étais pour le vieillard, Achuleta, ses enfants et ses petits enfants, et peut-être pour les enfants de ceux-ci sur la terre et dans le ciel, 'celui-qui-marchedans-le-soleil', parce que j'étais arrivé chez eux de l'Orient. (Retour..., 33-34)

Le poète se construit par et dans ses voyages, il se redonne une famille d'élection, qu'il refonde à sa manière. Le poète, l'homme, est fier de s'être fait donner un nom - et non de l'avoir imposé- par les Indiens : cela signifie qu'il est reconnu comme un des leurs. La pérégrination inscrite dans le nom (d'est en ouest) devient le signe d'une constitution de l'être en mouvement.

On pense à cet autre Lawrence, d'Arabie celui-là, qui lui aussi s'était fait arabe par amour et respect du peuple qui le recevait, ou encore à Rimbaud l'Ethiopien. Ce n'est pas un écrivain que viennent d'ondoyer les Indiens du Pueblos de Taos, c'est un homme comme eux, un Indien. Ce nom le relie à la terre, aux animaux et aux autochtones, autres " indiens ", c'est-à-dire aux peuples qui habitent et cultivent la terre. L'épisode est repris une troisième fois, sous une forme différente, dans Un Cimetière Indien avec le personnage de Maleterre, le bien nommé : "Maleterre a soudain le sentiment de se trouver avec ces indiens comme autrefois chez les paysans de sa terre natale ». (Un Cimetière..., 119) Temple résout ainsi sa double postulation : il réunit dans une seule image de l'autre ses oncles, à la fois sédentaires et voyageurs, amoureux de la terre, comme on peut l'être d'une femme, d'un amour panthéiste. 


\section{Conclusion}

Après ces longs voyages, après la réalisation de ses rêves d'enfance, après s'être retrouvé dans les Indiens, le poète revient définitivement en sa terre d'origine : "Voici le temps du retour aux herbages/ après les grandes fenaisons,/ le temps mémorial, du plain-chant/ de l'enfance, érigé sur les sources/ à l'orée du voyage.// Sous le lichen gît la pierre/ intacte; et les voix/ ranimées aux bruits des pas furtifs/ du vieil enfant bourdonnent/ dans le rucher panique/ des années convoquées. " (Anthologie..., 174) On dirait que le poète souhaite retrouver la permanence, que sa terre n'ait pas vieilli avec lui, qu'elle soit comme quand il était enfant : "Cinquante ans de soleils et d'averses [...]/ (Seul j'ai vieilli pour n'être pas resté) ". (Anthologie..., 176)

Temple est un poète de la terre et du soleil. Le soleil de son pays natal et celui des dieux indiens. C'est un poète profondément attaché à la littérature qui envahit ses textes, autant par les mots qui attestent une connaissance profonde de la terre, que par les auteurs qu'il a lus.

Son chant est d'une ampleur peu commune, surtout dans ces années attachées au bref. Ampleur tout aussi bien dans le sens de l'élévation. La vue du monde relativise la pensée et provoque un retour sur soi. L'être s'interroge sur son destin, son passé, rarement sur son avenir. Il découvre que ce qui importe dans le voyage, ce ne sont pas les souvenirs, ce sont les enseignements.

Il retiendra alors cette leçon donnée à Maleterre : "Si tu étais un véritable Indien tu désirerais mourir sur la terre de tes ancêtres ". (La Chasse infinie, 160) "J'ai quitté le mien pour retrouver les traces que mon enfance interrogeait comme celles d'un paradis perdu. Mais ces hommes, qui n'avaient jamais imaginé qu'ils puissent vivre ailleurs que sur le sol où s'affirmait la présence de leurs dieux, me disaient que j'avais pris la fausse piste ». (Un Cimetière..., 165) Si le poète qui est avec nous cette après-midi a retrouvé la piste de son enfance, nous savons maintenant qu'il en rapporte deux noms; " Dib, l'homme qui tue et pèle les chacals " et " celuiqui-marche-dans-le-soleil ». Deux noms qui soulignent les deux postulations de Temple : le sédentaire et le nomade. Ainsi baptisé "Oint du musc des troupeaux ", Frédéric Jacques Temple est un " arbre voyageur ". 